\title{
Fornax compact object survey FCOS: On the nature of Ultra Compact Dwarf galaxies
}

\author{
S. Mieske ${ }^{1,2}$, M. Hilker ${ }^{1}$, and L. Infante ${ }^{2}$ \\ 1 Sternwarte der Universität Bonn, Auf dem Hügel 71, 53121 Bonn, Germany \\ 2 Departamento de Astronomía y Astrofísica, P. Universidad Católica, Casilla 104, Santiago 22, Chile
}

Received 21 November 2003 / Accepted 16 January 2004

\begin{abstract}
The results of the Fornax Compact Object Survey (FCOS) are presented, complementing the first part of the FCOS described in Mieske et al. (2002). The FCOS aims at investigating the nature of the Ultra Compact Dwarf galaxies (UCDs) recently discovered in the center of the Fornax cluster (Drinkwater et al. 2000a). 280 unresolved objects with colours $0<$ $(V-I)<1.5 \mathrm{mag}$ in the magnitude space covering UCDs and bright globular clusters $(18<V<21 \mathrm{mag})$ were observed spectroscopically. 54 new Fornax members were found, plus five of the seven already known UCDs. Their distribution in radial velocity, colour, magnitude and space was investigated. It is found that bright compact objects $\left(V<20\right.$ or $\left.M_{V}<-11.4 \mathrm{mag}\right)$, including the UCDs, have a higher mean velocity than faint compact objects $(V>20 \mathrm{mag})$ at $96 \%$ confidence. The mean velocity of the bright compact objects is consistent with that of the dwarf galaxy population in Fornax, but inconsistent with that of NGC 1399's globular cluster (GC) system at 93.5\% confidence. The compact objects follow a colour magnitude relation with a slope very similar to that of normal dEs, but shifted about 0.2 mag redwards. The magnitude distribution of compact objects shows a fluent transition between UCDs and GCs. A slight overpopulation of $8 \pm 4$ objects for $V<20$ mag is detected with respect to the extrapolation of NGC 1399's GC luminosity function. The spatial distribution of bright compact objects is in comparison to the faint ones more extended at $88 \%$ confidence. All our findings are consistent with the threshing scenario (Bekki et al. 2003), suggesting that a substantial fraction of compact Fornax members brighter than $V \simeq 20$ mag could be created by threshing dE,Ns. Fainter than $V \simeq 20 \mathrm{mag}$, the majority of the objects seem to be genuine GCs. Our results are also consistent with merged stellar super-clusters (Fellhauer \& Kroupa 2002) as an alternative explanation for the bright compact objects.
\end{abstract}

Key words. galaxies: clusters: general - galaxies: dwarf - galaxies: fundamental parameters galaxies: kinematics and dynamics - galaxies: star clusters - Galaxy: globular clusters: general

\section{Introduction}

\section{1. "Normal" dwarf galaxies}

The faint end of the galaxy luminosity function is mainly populated by the dwarf elliptical galaxies (dE) and the dwarf spheroidals (dSph, the faintest dwarf galaxies in the Local Group). They are the most numerous type of galaxies in the nearby universe, having absolute magnitudes fainter than $M_{\mathrm{V}} \simeq-17 \mathrm{mag}$. They follow a tight magnitude-surface brightness $(M-\mu)$ relation in the sense that central surface brightness increases with increasing total luminosity (Ferguson \& Sandage 1988, 1989; Drinkwater et al. 2001a; Hilker et al. 2003). A consequence of this $M-\mu$ relation is that the size of dEs only changes very slowly with total luminosity, with the effective radius being of the order of a few hundred pc. For photometric studies of dEs in galaxy clusters this means that the approximate angular size of candidate dwarf galaxies is known.

Send offprint requests to: $\mathrm{S}$. Mieske, e-mail: smieske@astro.uni-bonn.de
This makes the morphological separation between cluster and back- or foreground galaxies easier.

\subsection{Ultra Compact Dwarf galaxies (UCDs)}

However, dwarf galaxies with sizes much smaller than normal dEs at comparable total luminosities are morphologically misclassified either as stars or distant background galaxies when using the $M-\mu$ relation as a membership criterion. In order to test the magnitude of such a bias, among other scientific tasks, Drinkwater et al. (2000a,b) performed the allobject Fornax Cluster Spectroscopic Survey (FCSS) down to $V \simeq 19.5\left(M_{V} \simeq-11.9\right) \mathrm{mag}$, using the $2 \mathrm{dF}$ spectrograph mounted on the Anglo-Australian Telescope. They were able to confirm the existence of a tight magnitude-surface brightness relation for the majority of the Fornax dwarf galaxies.

In addition they discovered a total of seven unresolved Fornax members, being much smaller than normal dwarf galaxies at comparable magnitudes (Drinkwater et al. 2000a, 2003; Karick et al. 2003). Two of the UCDs had first been discovered 
by Hilker et al. (1999). Drinkwater et al. named these objects "Ultra Compact Dwarf galaxies" (UCDs). They are located in the central projected $30^{\prime}$ of the Fornax cluster around NGC 1399 and cover a luminosity range of $18<V<19.5 \mathrm{mag}$, or $-13.4<M_{V}<-11.9 \mathrm{mag}((m-M)=31.4 \mathrm{mag}$, Ferrarrese et al. 2000). This makes them brighter than the brightest Milky Way globular cluster $\omega$ Centauri $\left(M_{V} \simeq-10.2\right.$ mag), but by far fainter than the compact dwarf elliptical galaxy M 32 $\left(M_{V} \simeq-16 \mathrm{mag}\right.$, see Graham 2002). The UCDs do fall into the luminosity range of dwarf galaxy nuclei (Lotz et al. 2001).

\subsection{Possible origins of UCDs}

Three different origins for the UCDs are in discussion: (1) they are the brightest globular clusters (GCs) of NGC 1399's very rich globular cluster system (GCS) (e.g. Mieske et al. 2002; Dirsch et al. 2003); (2) they are the remnant nuclei of stripped dwarf galaxies which have lost their outer parts in the course of tidal interaction with the Fornax cluster's potential (Bekki et al. 2003); (3) they are formed from the amalgamation of stellar super-clusters that were created in collisions between gas-rich galaxies (Fellhauer \& Kroupa 2002; Kroupa 1998; Maraston et al. 2004).

Regarding possibility (1): NGC 1399's GCS contains about 6500 GCs (about 60 times more than the Milky Way) and extends out to about 30' (Dirsch et al. 2003). The existence of genuine GCs in the same projected area and the same magnitude range as the UCDs cannot be ruled out a priori.

Regarding possibility (2): Bekki et al. (2001, 2003) show that threshing of "normal" dE,Ns in the Fornax cluster's central potential can create compact remnants with properties like the UCDs, given that the following requirements are fulfilled. The progenitor $\mathrm{dE}, \mathrm{N}$ needs to have a sufficiently high mass to partially survive the tidal disruption and a shallow dark matter core in order to allow substantial tidal disruption. In addition, its orbit needs to be very eccentric and its pericenter smaller compared to average cluster dEs. Therefore, the spatial distribution of the UCDs should be more concentrated towards the cluster center than that of the normal cluster dEs, which is qualitatively consistent with the radial distribution of the UCDs found by Drinkwater et al. Based on the simulations of Bekki et al. (2003 and private communications) this means that also the peculiar velocities of the progenitor dE,Ns with respect to the cluster's center of mass velocity would be limited to a smaller range than for average dEs. Bekki et al. conclude that due to these requirements, in the Fornax cluster the total number of UCDs should not be much higher than ten, covering a luminosity range of $-15<M_{V}<-8$ mag.

Regarding possibility (3): Fellhauer \& Kroupa (2002) have shown that in violent star forming regions, like dense knots in tidal arms of merger events, a conglomerate of young massive stellar clusters will rapidly merge to form a young stellar super cluster, which itself will turn into an object with similar properties as the UCDs after aging several Gyrs. Fellhauer \& Kroupa find that even for highly eccentric orbits, these compact star clusters maintain most of their mass after 10 Gyrs. Examples for young very massive conglomerates of stellar clusters are the star formation knots in the Antenna galaxies (Whitmore et al. 1999) and the star cluster W3 in NGC 7252 (Maraston et al. 2004). One possible origin for the UCDs could then be merger events several Gyrs ago in the Fornax cluster which led to the creation of massive super-clusters.

\subsection{High resolution follow up investigations of UCDs}

Follow-up high resolution photometry with the HST-STIS and high resolution UVES spectroscopy (Drinkwater et al. 2003) showed that the UCDs have effective radii between 10 and $22 \mathrm{pc}$ and internal velocity dispersions between 24 and $37 \mathrm{~km} \mathrm{~s}^{-1}$, placing them at the extreme end of what is found for globular clusters (Djorgovski et al. 1997; Meylan et al. 1994, 2001). Their $M / L_{V}$ ratios are between 2 and 4 , in the same range as derived for the very luminous stellar cluster Mayall II in M 31 by Meylan et al. (2001) and for $\omega$ Centauri (Meylan 1987; Meylan et al. 1994, 1995), but higher than for the vast majority of GCs, which have values of $M / L_{V} \simeq 1$. Note that both Mayall II and $\omega$ Centauri are very bright stellar clusters, and are in general not even considered as normal GCs due to their complex star formation history (Hilker \& Richtler 2000). The simulations of Bekki et al. predict $M / L_{V}$ ratios for the threshed nuclei in the same range as observationally found for the UCDs.

Drinkwater et al. (2003) find that in the velocity dispersionabsolute magnitude $\left(\sigma-M_{V}\right)$ plane, the UCDs lie off the extrapolation of the relation defined by the GCs and rather appear to follow the Faber-Jackson relation for elliptical galaxies (Faber \& Jackson 1976). They populate the same region as dE-nuclei. However, as this disagreement of the UCD values is only with respect to the extrapolation of the GC relation defined at fainter magnitudes, it is not a sufficient condition to separate UCDs from GCs. This would require $\sigma$ measurements of bona fide GCs in the brightness range of UCDs.

In conclusion, if the UCDs were bright genuine GCs, they would lie at the extreme end of the mass-light-size space of their kind (Drinkwater et al. 2003), while if they were nuclei of stripped dE,Ns (Bekki et al. 2003) or merged stellar superclusters (Fellhauer \& Kroupa 2002), they would be more average examples of their kind.

\subsection{The first part of the "Fornax Compact Object Survey" FCOS}

Although the results obtained from high-resolution spectroscopy and photometry suggest that the UCDs are more complicated objects than normal GCs, no definite discriminating judgment on their nature has been made until now. Therefore, investigating group properties of bright compact Fornax members like their distribution in radial velocity, magnitude, colour and space can prove to be a very valuable contribution to the ongoing discussion ${ }^{1}$. It would be very interesting to know whether the compact Fornax members are divided up into

\footnotetext{
With compact, we here refer to sources unresolved on ground based imaging, corresponding to an upper limit of a few tens of parsecs in radius at the Fornax cluster distance.
} 
subgroups with respect to any of these four global properties. Do the fainter ones behave more like GCs, while the brighter ones (including the UCDs) are different?

As Drinkwater et al. (2000a,b) were not able to close the magnitude gap towards the GCs due to the design of their survey, the approach of our investigation is to look for compact Fornax members in the magnitude regime between the UCDs and bright GCs, including both groups of objects. To this end, we have conducted the Fornax Compact Object Survey (FCOS), whose first part (FCOS-1) was described in Mieske et al. (2002, hereafter Paper I). In FCOS-1, about $20 \%$ of compact Fornax candidates within 20' of NGC 1399 were observed spectroscopically. 12 new compact Fornax members in the magnitude regime $19.7<V<21 \mathrm{mag}$ were found. The resulting luminosity distribution, shown in Fig. 5 of Paper I, supported a fluent transition between UCDs and GCs, speaking against a magnitude gap between both populations. However, the number counts involved were too low to give a more quantitative statement. With only 12 objects at hand, also the velocity, colour and spatial distribution of the compact Fornax members was too poorly sampled to investigate possible segregations into subgroups.

\subsection{Aim of this paper}

In this paper we present FCOS-2, the second part of our survey, and merge the datasets of FCOS- 1 and FCOS-2. FCOS-2 increases the area coverage of FCOS- 1 by a factor of about three. There are four main aspects that are addressed with the entire FCOS sample at hand:

1. Investigate the radial velocity distribution in order look for dynamically distinct subgroups;

2. determine the luminosity distribution of compact Fornax members in order to check whether a significant number of stripped dE-nuclei mixes up with the bright GCs;

3 . investigate the colour-magnitude $(\mathrm{CM})$ relation of compact Fornax members in order to compare it both with the colours of GCs and dwarf galaxies;

4. investigate the radial distribution of compact Fornax members in order to look for differences in the spatial distribution between subgroups.

The paper is structured as follows: in the next section, the candidate selection and the observations for FCOS-2 are described. In Sect. 3, the data reduction is described. The results of the entire FCOS are shown in Sect. 4, based on the merging of the FCOS-1 and FCOS-2 database. The results are discussed in Sect. 5. We finish the paper with summary and conclusions in Sect. 6.

\section{Candidate selection and observations}

To select candidates for FCOS-2, we used two different image sets with the aim to optimize our spatial coverage.

First, the same $25^{\prime} \times 25^{\prime}$ wide field images used in FCOS-1, obtained in Johnson $V$ and $I$ with the $2.5 \mathrm{~m}$ Du Pont Telescope at Las Campanas Observatory, Chile, in December 1999 (see Paper I and Hilker et al. 2004, in prep. for more details).

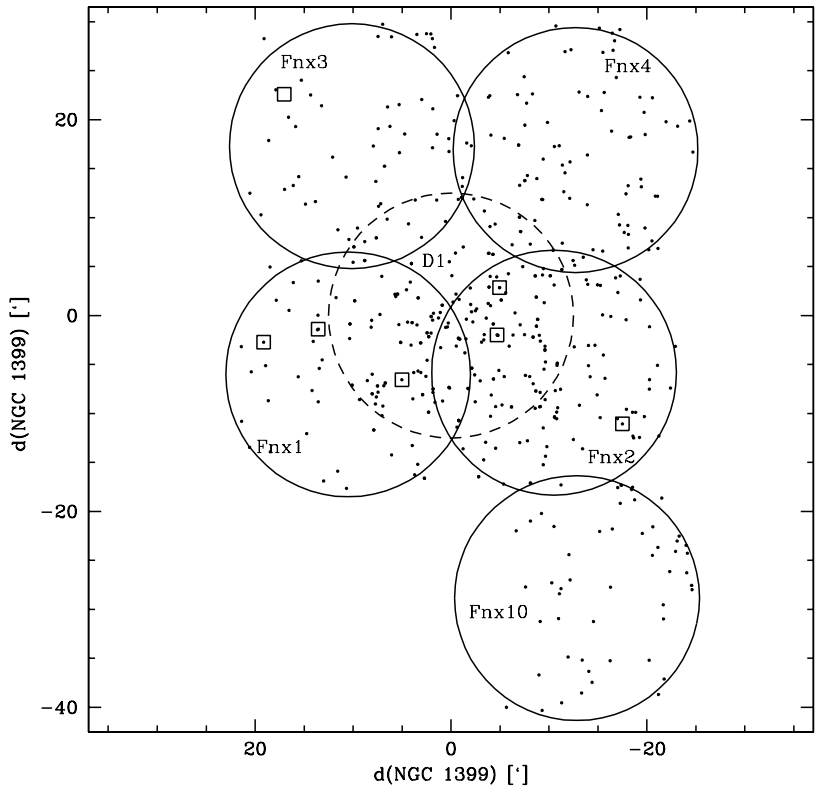

Fig. 1. Map of the central Fornax cluster, with coordinates centered on NGC 1399. Dots indicate the positions of all previously unobserved candidates satisfying our photometric selection criteria for possible globular clusters. Squares indicate the position of the 7 UCDs. Solid circles indicate the approximate field limits of the LCO-images and mask regions, which in reality are not perfectly circular. The dashed circle indicates the field where additional candidates were chosen from the CTIO-data (Dirsch et al. 2003). The UCD in field Fnx3 (UCD 5) was not classified as unresolved by SExtractor, being given a starclassifier value of 0.18 in $V$ and 0.23 in $I$. Note that also UCD 3 in Fnx1 is given low star classifier values of 0.46 in $V$ and 0.36 in $I$, just above the minimum value of 0.45 required to satisfy our selection criteria.

Second, photometric data from Dirsch et al. (2003, and private communications) obtained in the Washington $C$ and Kron $R$ filters with the $36^{\prime} \times 36^{\prime}$ MOSAIC camera at the $4 \mathrm{~m}$ CTIO telescope.

The CTIO-data cover the entire central region of NGC 1399, allowing the selection of candidates very close to its center. This was not possible with the LCO-data as they miss out much of the bright central region of NGC 1399.

In Fig.1, the location of the LCO- and CTIO-fields is indicated.

\subsection{Selection of candidates from the LCO photometry}

In contrast to Paper I, we now selected only unresolved objects, defined by the SExtractor star classifier (Bertin \& Arnouts 1996) being larger than 0.45 either in $V$ or in $I$. This was done because in Paper I $100 \%$ of the extended objects investigated spectroscopically turned out to be background galaxies far behind the Fornax cluster. Of the unresolved objects, only those were selected which had not been observed either in the FCSS nor in the FCOS before and which satisfy the conditions $V<21 \mathrm{mag}$ and $(V-I)<1.40$. The magnitude limit is identical to Paper I, being about 3 mag brighter than the GCLF turn-over and about $1.5 \mathrm{mag}$ fainter than the faintest UCD. The red colour limit is 0.10 mag bluer than the 1.50 mag adopted in Paper I in 
order to decrease contamination by non-cluster members, as the reddest newly discovered Fornax member from Paper I had $(V-I)=1.27 \mathrm{mag}$.

\subsection{Selection of candidates from the CTIO photometry}

As for the LCO-images, only objects unresolved on the CTIO-data (Dirsch et al. 2003) were regarded as possible candidates. The CTIO-data had been obtained in the Washington $C$ and Cron $R$ filters, in contrast to our Johnson $V$ and $I$ data from LCO. The magnitude limit of $V<21 \mathrm{mag}$ was transformed into $R<20.5 \mathrm{mag}$, with $(V-R)=0.5 \mathrm{mag}$ taken from Worthey (1994) for a typical GC population of 12 Gyrs and $[\mathrm{Fe} / \mathrm{H}]=-1 \mathrm{dex}$. The colour window was adopted as $1.0<$ $(C-R)<2.0$, based on the results of Dirsch et al. (2003). This range corresponds approximately to $0.8<(V-I)<1.3 \mathrm{mag}$ (Dirsch, private communications), extending to about $0.1 \mathrm{mag}$ redder than the colour range of UCDs, see Table 1 of Paper I or Fig. 10 of this paper. Therefore, it is unlikely that any UCD is missed by applying this selection window.

It holds that $(C-R)-(V-I)=0.5 \mathrm{mag}$, according to Worthey (1994), for the same typical GC population with $(V-R)=0.5$.

In total, 462 unresolved candidate objects in the central Fornax cluster were selected for the FCOS, covering a colourmagnitude range of $0<(V-I)<1.5$ and $18<V<21$ mag.

\subsection{Observations}

The observations for FCOS-2 were performed in the three nights of 2002/12/04 to 2002/12/06 at the $2.5 \mathrm{~m} \mathrm{Du}$ Pont telescope at Las Campanas. The instrument was the Wide Field CCD (WFCCD) camera, which reimages a $25^{\prime}$ field onto a Tek\#5-Detector of $2048 \times 2048$ pixel with a scale of 0.774 "/pixel. Multi-slit masks and the "blue" grism, which has a transmission between 3600 and $7800 \AA$ and a dispersion of about $3 \AA$ per pixel, were used. As the slit width in our observation was $\approx 1.5^{\prime \prime}$ (=2 pixel), the effective resolution was of the order of about $6 \AA$, corresponding to $450 \mathrm{~km} \mathrm{~s}^{-1}$ at $4000 \AA$.

We did not use the higher resolution " $\mathrm{H} \& \mathrm{~K}$ " grism used in FCOS-1, as the spectral resolution achieved in FCOS-1 was not sufficient to study possible line index differences between UCDs and bright GCs (Paper I). The "blue" grism with its lower resolution and higher wavelength coverage allowed smaller integration times.

The locations of the candidates for each field are indicated in Fig. 1. There is some overlap between the central field D1 and the adjacent fields Fnx1 to Fnx4. Measurements of the same object in different fields are used in Sect. 3.1 for estimating the systematic uncertainties in radial velocity determination. A slit could not be allocated on the mask for all candidates (see Fig. 3), as a minimum slit-length of 9 arcsec and a minimum distance between neighbouring candidates of 3 arcsec perpendicular to the dispersion direction was demanded.

A total of six fields as indicated in Fig. 1 was observed in the three nights, using eight different slit masks (three masks for D1, the field with the highest candidate density). The total integration time per mask was $2 \mathrm{~h}$. The spectra were calibrated with lamp exposures taken directly before or after the science exposure. The same radial velocity standards as in FCOS-1 were observed in the course of the three nights.

\section{Data reduction}

Data reduction was performed with the IRAF-packages IMRED, TWODSPEC and ONEDSPEC. The mask-exposures were bias corrected and combined using a cosmic-ray removal algorithm. The combined images were response calibrated using the dome-flat spectra averaged perpendicularly to the dispersion direction. Then, each single 2D science spectrum including the corresponding sky region and the calibration lamp exposures were cut out of the entire image. The spectra were extracted using the task APALL in the ONEDSPEC package and then wavelength calibrated with the task IDENTIFY in the ONEDSPEC package.

The radial velocity of the observed objects was determined with cross-correlation, applying the IRAF-task FXCOR in the $\mathrm{RV}$-package. The same three objects as in FCOS-1 were used as radial velocity templates for the cross-correlation: the reobserved spectra of HD 54810 and HD 22879, and a synthetic spectrum taken from Quintana et al. (1996). The mean of the three cross correlations, corrected for the respective heliocentric velocities of the templates, was adopted as the final radial velocity.

\subsection{Systematic radial velocity errors}

\subsubsection{Errors from double observations within FCOS}

The overlap between the central field D1 and the adjacent fields resulted in a total of ten objects that were observed spectroscopically twice. These double measurements allow an estimate of the systematic uncertainty of the radial velocity results. See top panel of Fig. 2 for the corresponding plot. The mean single measurement error is $90 \mathrm{~km} \mathrm{~s}^{-1}$. The median difference between D1 from FCOS-2 and FCOS-1 is $85 \pm 34 \mathrm{~km} \mathrm{~s}^{-1}$, while the two objects observed twice in FCOS-2 agree nicely. This shows that there is good consistency between the masks used in FCOS-2, but that there is a systematic difference between FCOS- 1 and FCOS-2 of the order of the single measurement error. A difference of $100 \mathrm{~km} \mathrm{~s}^{-1}$ corresponds to a shift of about $1.5 \AA$, or half a pixel with the blue grism and about one pixel with the H\&K grism. The slit width is about 2 pixel in FCOS-1 and FCOS-2. Thus, the order of this systematic difference is about $25-50 \%$ of the slit-width and therefore within the expectable error range, possibly caused by instrument flexion.

\subsubsection{Errors from comparison with radial velocities from Dirsch et al.}

As an additional test, we compare our radial velocities with those observed by Dirsch et al. (2004, private communications), who have obtained about 500 spectra of GCs around 
Table 1. All Fornax cluster members detected in the FCOS, ordered by magnitude. The object codes are "FCOS x-xxx" for FCOS-1 (Mieske et al. 2002) and "FCOS x-xxxx" for FCOS-2 (this paper). Errors in $V$ and $(V-I)$ are between 0.04 and 0.05 mag, as judged from the artificial star experiments described in Sect. 4.2.1. * For all objects FCOS 0-2xxx, $V$ and $(V-I)$ are derived indirectly from the $C-R$ photometry of Dirsch et al. (2003), using $V=R+0.5 \mathrm{mag}$ and $(V-I)=(C-R)-0.5 \mathrm{mag}$ (see text for further details). ${ }^{* *} v_{\text {rad }}$ is the mean of the values measured in FCOS-1 and FCOS-2 (see text for further details).

\begin{tabular}{|c|c|c|c|c|c|c|c|}
\hline Name & $\overline{\alpha \alpha(2000.0)}$ & 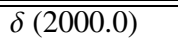 & $v_{\mathrm{rad}}$ & $\overline{\overline{\Delta \Delta v_{\mathrm{rad}}}}$ & 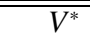 & $(V-I)^{*}$ & Comments \\
\hline FCOS 1-2053 & $03: 38: 54.05$ & $-35: 33: 33.8$ & 1493 & 61 & 18.06 & 1.20 & UCD 3 \\
\hline FCOS 2-2143 & 03:38:05.04 & $-35: 24: 09.7$ & 1244 & 55 & 18.93 & 1.16 & UCD 6 \\
\hline FCOS 1-2083 & 03:39:35.90 & $-35: 28: 24.2$ & 1848 & 85 & 19.12 & 1.14 & UCD 4 \\
\hline FCOS 2-2111 & 03:38:06.29 & $-35: 28: 58.8$ & 1280 & 58 & 19.23 & 1.14 & UCD 2 \\
\hline FCOS 2-2031 & 03:37:03.24 & $-35: 38: 04.6$ & 1571 & 75 & 19.31 & 1.17 & UCD 1 \\
\hline FCOS $1-021$ & 03:38:41.96 & $-35: 33: 12.9$ & 2010 & 40 & 19.70 & 1.18 & \\
\hline FCOS 3-2004 & $03: 39: 20.50$ & $-35: 19: 14.2$ & 1341 & 57 & 19.73 & 1.23 & \\
\hline FCOS 3-2027 & $03: 38: 23.74$ & $-35: 13: 49.4$ & 1553 & 72 & 19.76 & 1.04 & \\
\hline FCOS 0-2031 & 03:38:28.97 & $-35: 22: 55.9$ & 1654 & 69 & 19.87 & 1.13 & \\
\hline FCOS 2-2134 & 03:38:10.73 & $-35: 25: 46.2$ & 1683 & 86 & 19.90 & 1.34 & \\
\hline FCOS $0-2030$ & $03: 38: 28.34$ & $-35: 25: 38.3$ & 1771 & 56 & 20.01 & 1.10 & \\
\hline FCOS $2-2153$ & 03:38:06.48 & $-35: 23: 03.8$ & 1390 & 124 & 20.04 & 1.08 & \\
\hline FCOS 1-2024 & $03: 38: 25.54$ & $-35: 37: 42.6$ & 1789 & 124 & 20.06 & 1.20 & \\
\hline FCOS 0-2066 & $03: 38: 23.23$ & $-35: 20: 00.6$ & 1255 & 69 & 20.09 & 1.12 & \\
\hline FCOS 1-060 & 03:39:17.66 & $-35: 25: 30.0$ & 980 & 45 & 20.19 & 1.27 & \\
\hline FCOS 0-2033 & 03:38:30.72 & $-35: 27: 46.1$ & 1400 & 113 & 20.28 & 1.29 & \\
\hline FCOS $1-063^{* *}$ & 03:38:56.14 & $-35: 24: 49.1$ & 688 & 45 & 20.29 & 1.05 & \\
\hline FCOS 0-2024 & 03:38:16.51 & $-35: 26: 19.3$ & 902 & 93 & 20.32 & 0.74 & \\
\hline FCOS 2-073 & 03:38:11.98 & $-35: 39: 56.9$ & 1300 & 45 & 20.40 & 1.20 & \\
\hline FCOS 4-2028 & 03:37:43.49 & $-35: 15: 10.1$ & 1240 & 89 & 20.41 & 1.18 & \\
\hline FCOS 3-2019 & 03:39:37.18 & $-35: 15: 22.0$ & 1921 & 114 & 20.49 & 1.10 & \\
\hline FCOS 2-2106 & 03:38:25.06 & $-35: 29: 25.1$ & 1313 & 168 & 20.51 & 0.99 & \\
\hline FCOS 2-2107 & $03: 38: 25.66$ & $-35: 29: 19.7$ & 1267 & 101 & 20.51 & 1.32 & \\
\hline FCOS 0-2007 & $03: 38: 54.67$ & $-35: 29: 44.2$ & 1761 & 98 & 20.54 & 0.91 & \\
\hline FCOS 2-2161 & 03:37:33.86 & $-35: 22: 19.2$ & 2009 & 106 & 20.54 & 1.02 & \\
\hline FCOS 2-2165 & $03: 37: 28.22$ & $-35: 21: 23.0$ & 1356 & 139 & 20.57 & 0.97 & \\
\hline FCOS 1-019 & 03:38:54.59 & $-35: 29: 45.8$ & 1680 & 35 & 20.62 & 1.01 & \\
\hline FCOS 1-2095 & 03:38:33.82 & $-35: 25: 57.0$ & 1245 & 220 & 20.66 & 1.09 & \\
\hline FCOS 0-2023 & 03:38:12.70 & $-35: 28: 57.0$ & 1705 & 76 & 20.66 & 1.11 & \\
\hline FCOS 0-2062 & 03:38:18.43 & $-35: 27: 39.6$ & 1338 & 81 & 20.67 & 0.89 & \\
\hline FCOS $1-058^{* *}$ & 03:38:39.30 & $-35: 27: 06.4$ & 1661 & 60 & 20.67 & 1.04 & \\
\hline FCOS 0-2069 & $03: 38: 26.71$ & $-35: 30: 07.2$ & 1914 & 91 & 20.67 & 0.99 & \\
\hline FCOS 2-078 & $03: 37: 41.83$ & $-35: 41: 22.2$ & 1025 & 60 & 20.69 & 1.21 & \\
\hline FCOS 2-2094 & $03: 37: 42.24$ & $-35: 30: 33.8$ & 1462 & 121 & 20.71 & 1.16 & \\
\hline FCOS 0-2072 & 03:38:32.06 & $-35: 28: 12.7$ & 1559 & 102 & 20.71 & 1.16 & \\
\hline FCOS 2-2072 & 03:38:14.69 & $-35: 33: 40.7$ & 1331 & 149 & 20.72 & 0.91 & \\
\hline FCOS 0-2041 & 03:38:44.11 & $-35: 19: 01.6$ & 1287 & 66 & 20.73 & 1.32 & \\
\hline FCOS $1-2103$ & 03:38:57.38 & $-35: 24: 50.8$ & 896 & 94 & 20.74 & 1.18 & \\
\hline FCOS 0-2063 & 03:38:19.08 & $-35: 26: 37.3$ & 1692 & 104 & 20.80 & 1.00 & \\
\hline FCOS $0-2032$ & $03: 38: 30.22$ & $-35: 21: 31.0$ & 1402 & 73 & 20.80 & 0.86 & \\
\hline FCOS 2-086 & 03:37:46.77 & $-35: 34: 41.7$ & 1400 & 50 & 20.81 & 0.92 & \\
\hline FCOS 0-2027 & 03:38:19.49 & $-35: 25: 52.3$ & 1289 & 130 & 20.83 & 1.43 & \\
\hline FCOS 0-2089 & 03:38:17.09 & $-35: 26: 30.8$ & 1294 & 95 & 20.83 & 1.16 & \\
\hline FCOS 2-2127 & 03:38:11.69 & $-35: 27: 16.2$ & 1476 & 201 & 20.83 & 1.18 & \\
\hline FCOS 1-2077 & 03:38:40.56 & $-35: 29: 10.0$ & 586 & 91 & 20.84 & 1.16 & \\
\hline FCOS $1-2115$ & 03:38:49.18 & $-35: 21: 42.1$ & 872 & 95 & 20.85 & 1.22 & \\
\hline FCOS 4-049 & 03:37:43.09 & $-35: 22: 12.9$ & 1330 & 50 & 20.85 & 0.98 & \\
\hline FCOS 2-089 & 03:38:14.02 & $-35: 29: 43.0$ & 1235 & 45 & 20.87 & 1.08 & \\
\hline FCOS 0-2025 & 03:38:17.98 & $-35: 15: 06.1$ & 1401 & 264 & 20.87 & 1.07 & \\
\hline FCOS 0-2026 & 03:38:19.03 & $-35: 32: 22.2$ & 1726 & 97 & 20.91 & 1.11 & \\
\hline FCOS 0-2074 & 03:38:35.66 & $-35: 27: 15.5$ & 2274 & 112 & 20.91 & 1.07 & \\
\hline FCOS 1-2089 & 03:38:48.86 & $-35: 27: 43.9$ & 1559 & 158 & 20.92 & 1.02 & \\
\hline FCOS 2-2100 & 03:38:00.17 & $-35: 30: 08.3$ & 997 & 152 & 20.94 & 1.07 & \\
\hline FCOS 2-095 & $03: 37: 46.55$ & $-35: 28: 04.8$ & 1495 & 45 & 20.96 & 1.14 & \\
\hline FCOS 1-2080 & 03:38:41.35 & $-35: 28: 46.6$ & 1647 & 124 & 20.96 & 0.99 & \\
\hline FCOS 1-064 & 03:38:49.77 & $-35: 23: 35.6$ & 900 & 85 & 20.96 & 1.21 & \\
\hline FCOS $1-2050$ & 03:39:19.06 & $-35: 34: 07.0$ & 1635 & 104 & 20.96 & 1.02 & \\
\hline FCOS 0-2092 & 03:39:05.02 & $-35: 26: 53.9$ & 970 & 205 & 20.96 & 1.14 & \\
\hline FCOS 0-2065 & $03: 38: 21.84$ & $-35: 29: 23.3$ & 1804 & 125 & 20.98 & 1.22 & \\
\hline
\end{tabular}



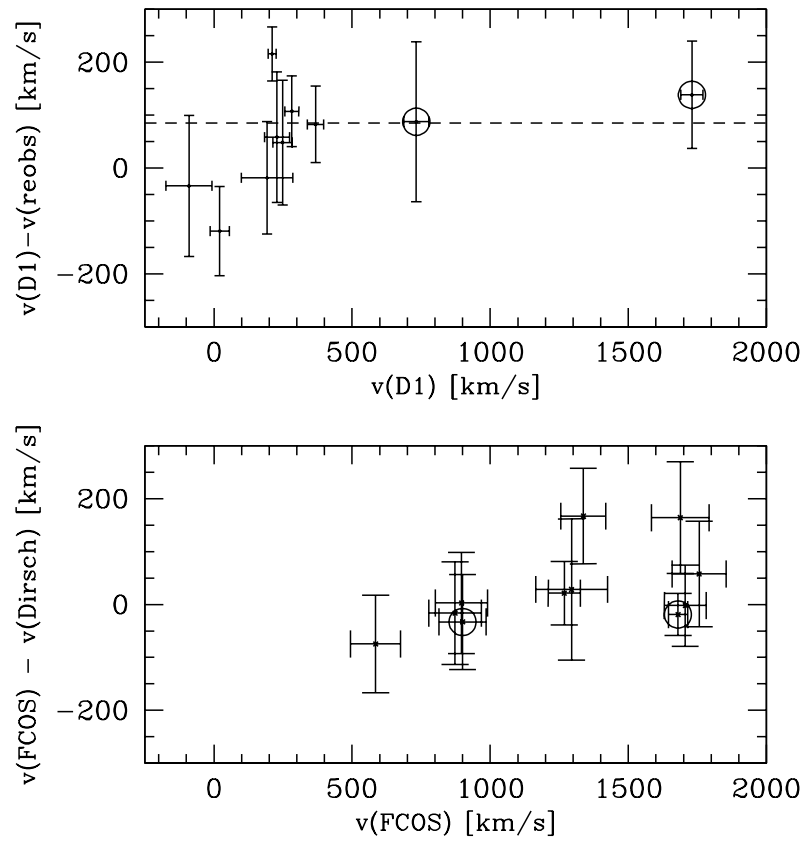

Fig. 2. Top panel: comparison between the two radial velocity measurements obtained in the FCOS for the objects that were observed twice due to the overlap of the central field D1 with adjacent fields. The $x$-axis shows the radial velocity obtained in field D1, during FCOS-2. The $y$-axis shows the difference between the D1-velocity and the velocity obtained in the adjacent field. The objects marked with circles were observed first in FCOS-1, the remaining ones in FCOS-2. The dashed line indicates the median difference between D1- and FCOS-1-velocities. Bottom panel: comparison between the FCOS radial velocities and the radial velocities from Dirsch et al. (private communication) for the objects observed in common. The two objects marked with circles are from FCOS-1, the rest from FCOS-2.

NGC 1399 brighter than $V \simeq 23 \mathrm{mag}$, with $20 \mathrm{GCs}$ in the same magnitude regime as the FCOS. They used FORS2/MXU at the VLT. See bottom panel of Fig. 2 for the corresponding plot. The mean difference between our and their radial velocity measurements for the eleven objects observed in common is $27 \pm 23 \mathrm{~km} \mathrm{~s}^{-1}$, almost consistent with zero. The scatter about the mean difference is $77 \mathrm{~km} \mathrm{~s}^{-1}$, the mean single measurement error of our results is $86 \mathrm{~km} \mathrm{~s}^{-1}$. This shows again that the errors resulting from our cross-correlation are a very good estimate of the real radial velocity uncertainty. Fitting a linear relation to the observed differences yields a slope different from zero at only the $0.45 \sigma$ significance, indicating that velocity difference is not correlated with velocity within our velocity errors. If one excludes the two FCOS-1 objects from the analysis, taking only FCOS-2 measurements, the mean radial velocity difference becomes $37 \pm 27 \mathrm{~km} \mathrm{~s}^{-1}$.

In conclusion, the radial velocities measured in FCOS-2 are about $85 \mathrm{~km} \mathrm{~s}^{-1}$ higher than in FCOS-1 and about $30-40 \mathrm{~km} \mathrm{~s}^{-1}$ higher than those from Dirsch et al. In other words, the values from Dirsch lie between the values from FCOS-1 and FCOS-2.

As a consequence, we adopt the mean of the FCOS-1 and FCOS-2 velocity for the two Fornax members observed twice in the course of the FCOS.

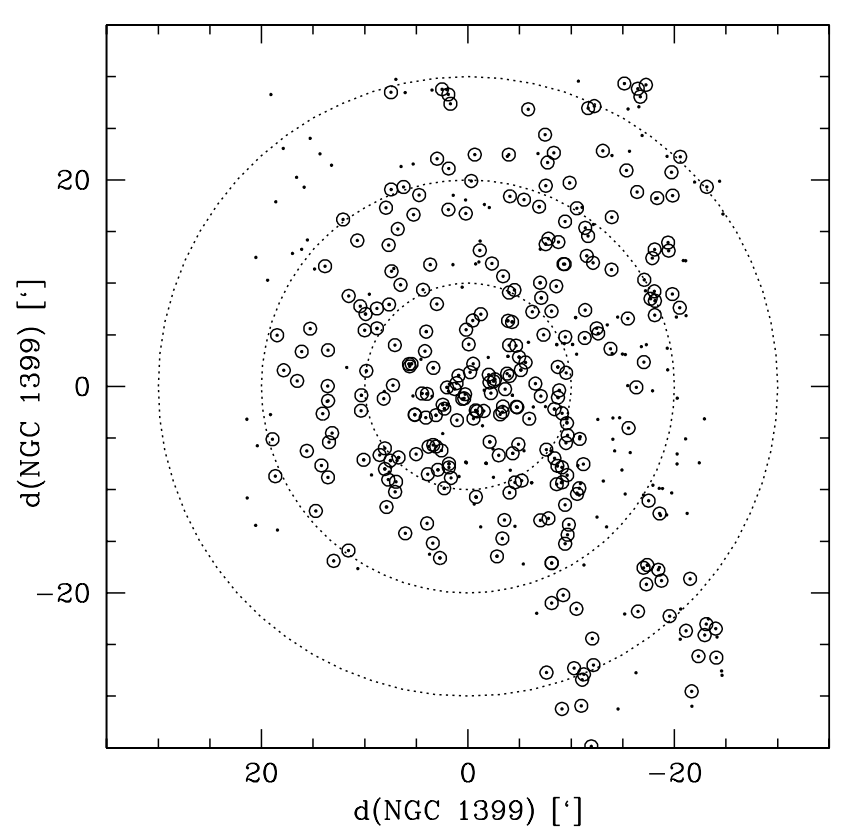

Fig. 3. Map of the central square degree around NGC 1399. Dots are all candidates for spectroscopic investigation that satisfy the selection criteria for FCOS as defined in Sects. 2.1 and 2.2. Small circles indicate the objects observed in the FCOS. The large dotted circles indicate distances of 10, 20 and 30 arcmin from NGC 1399.

\section{Results}

In the following subsections, the results of FCOS are presented, merging the smaller FCOS-1 data set with the larger one from FCOS-2. The total number of objects with successfully determined radial velocity in the FCOS is 280 - out of 462 candidates -, covering a colour-magnitude range of $0<(V-I)<1.5$ and $18<V<21 \mathrm{mag}$. To separate Fornax members from Milky Way stars and background galaxies, we applied a lower limit of $550 \mathrm{~km} \mathrm{~s}^{-1}$ and an upper limit of $2400 \mathrm{~km} \mathrm{~s}^{-1}$ in radial velocity for Fornax membership (see Fig. 5), resulting in 59 Fornax members out of the 280 observed objects. In Table 1, the 59 Fornax cluster members are tabulated. They have colours $0.8<(V-I)<1.4$, consisting of 12 members from FCOS-1, 42 new members from FCOS-2 and five of the seven already known UCDs. A list of all investigated objects in FCOS-2, including foreground stars and background galaxies, will be added to the existing list of FCOS- 1 objects at the CDS.

A map overplotting all candidates and observed candidates is given in Fig. 3. A map showing only the observed objects plus the Fornax members marked is given in Fig. 4.

\subsection{Radial velocity distribution}

Figure 5 shows the velocity histogram of the objects observed in FCOS. The mean velocity of the Fornax sample is $1425 \pm$ $45 \mathrm{~km} \mathrm{~s}^{-1}$, with a velocity dispersion of $326_{-32}^{+42} \mathrm{~km} \mathrm{~s}^{-1}$. Note that here and in what follows, the velocity dispersion is given by the quadratic difference of the standard deviation of the measured velocities around their mean and the mean velocity error. The standard deviation for the FCOS sample is $14 \mathrm{~km} \mathrm{~s}^{-1}$ 


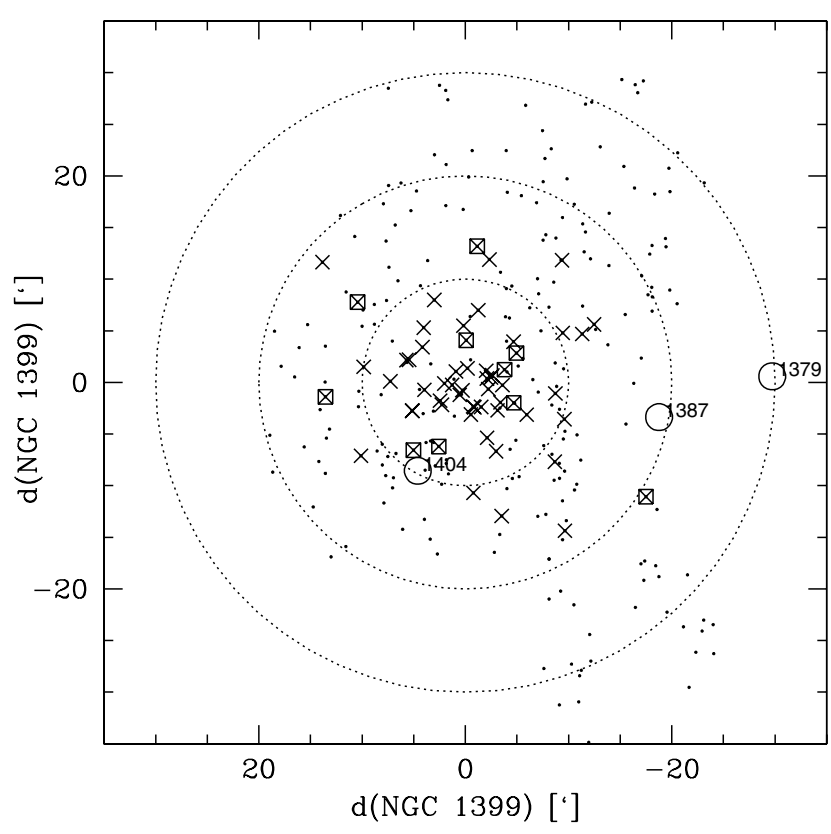

Fig. 4. Map of the same region as in Fig. 3. Dots are all objects observed in the FCOS, marked with small circles in the previous Fig. 3. Crosses indicate the observed objects with radial velocities in the Fornax cluster range, i.e. between 550 and $2400 \mathrm{~km} \mathrm{~s}^{-1}$. Squares mark the Fornax members brighter than $V=20$ mag. Circles mark the location of Fornax giant galaxies with their NGC numbers indicated except for NGC 1399, which is located in the origin.

higher than the velocity dispersion, with the mean velocity error being $97 \mathrm{~km} \mathrm{~s}^{-1}$.

Applying a Gaussian fit to the velocity histogram, the parameters are $v_{\text {rad }, 0}=1438 \pm 56 \mathrm{~km} \mathrm{~s}^{-1}, \sigma_{v}=372 \pm 46 \mathrm{~km} \mathrm{~s}^{-1}$. Although the overall velocity histogram for FCOS Fornax members in Fig. 5 is symmetric and well approximated by a Gaussian, Fig. 6 shows that objects brighter than $V=20$ mag appear to have a higher mean velocity than the faint ones and a slightly smaller velocity dispersion.

Defining $V=20 \mathrm{mag}$ as the limit between bright and faint Fornax members, a Kolmogorov-Smirnov test shows that the bright and faint velocity distribution are not drawn from the same underlying distribution at $74 \%$ confidence. To investigate this in more detail, we calculate the mean velocities and velocity dispersions for the bright and faint objects separately. These calculations are performed not only for the entire FCOSsample, but also for two modified samples:

- We first correct FCOS-1 and FCOS-2 for the systematic velocity difference found in Sect. 4.1 by adding $50 \mathrm{~km} \mathrm{~s}^{-1}$ to the FCOS-1 values and subtracting $35 \mathrm{~km} \mathrm{~s}^{-1}$ from the FCOS-2 values. The latter correction serves to remove the influence of systematic velocity errors on the velocity distributions, especially when considering the bright and faint sample separately;

- second, we add to the shift corrected FCOS sample the eight additional radial velocities from Dirsch et al. obtained for objects not observed in the FCOS and the velocities of the two UCDs not observed in the FCOS

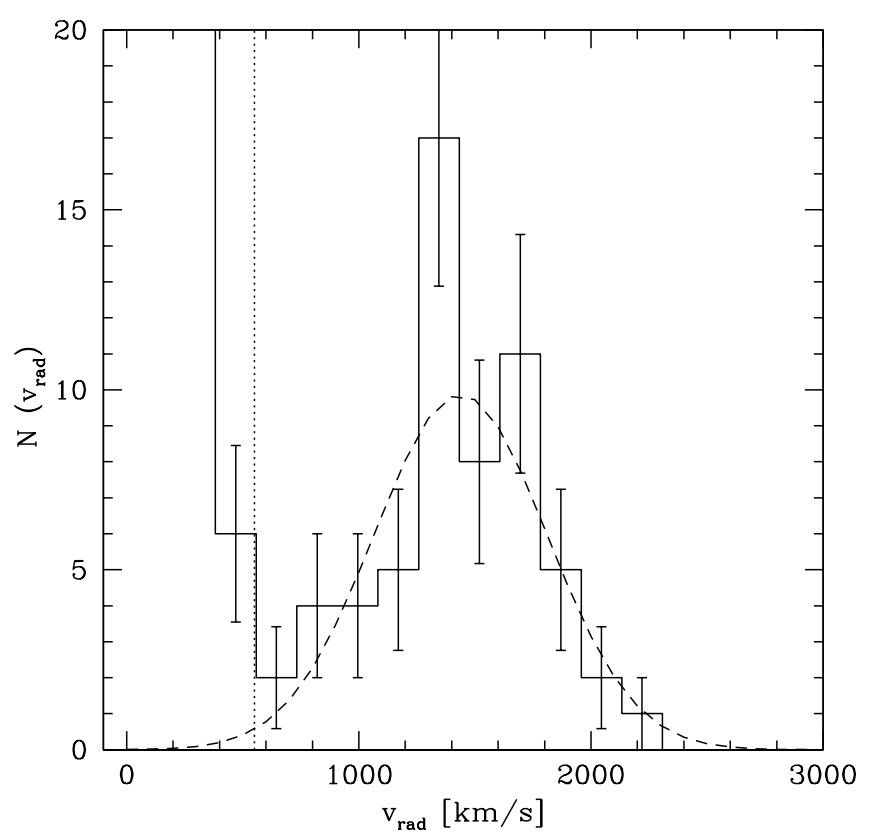

Fig. 5. Velocity histogram of the objects observed in the FCOS. The lower limit of $550 \mathrm{~km} \mathrm{~s}^{-1}$ applied for Fornax membership is indicated by the dotted line. The dashed line is a Gaussian fit to the histogram for velocities higher than $550 \mathrm{~km} \mathrm{~s}^{-1}$. Parameters are: $v_{\text {rad, }, 0}=1438 \pm$ $56 \mathrm{~km} \mathrm{~s}^{-1}, \sigma_{v}=372 \pm 46 \mathrm{~km} \mathrm{~s}^{-1}$.

(Karick et al. 2003). This latter sample will hereafter be referred to as shift corrected enlarged sample.

The Kolmogorov-Smirnov test yields $74 \%$ disagreement between bright and faint ones for the shift corrected sample and $88.8 \%$ disagreement for the shift corrected enlarged sample. The respective velocity histograms are shown in Fig. 7. The results of the calculations are shown in Table 2. One can see that the difference in mean velocity between the bright and faint subsample is over $200 \mathrm{~km} \mathrm{~s}^{-1}$ for the shift corrected enlarged sample. This is significant at the $96 \%(2.1 \sigma)$ confidence level, as derived from a t-test. The velocity dispersion is only marginally smaller for the bright objects, with the difference disappearing for the shift corrected enlarged sample.

We therefore conclude that we have found strong indications for the bright FCOS Fornax members having a higher mean velocity than the faint ones, while the velocity dispersions of both sub-samples differ insignificantly. The implications of these findings with respect to the nature of bright compact objects in Fornax will be discussed in Sect. 5, where the results will also be compared with results of other authors.

The following two expressions will be used from now on to distinguish between the bright and faint sample: "bright compact objects", referring to FCOS Fornax members with $V<20 \mathrm{mag}$; and "faint compact objects", referring to FCOS Fornax members with $V>20$ mag. With "FCOS objects", we will refer ourselves to all FCOS Fornax members.

\subsection{Magnitude distribution}

In this subsection, we investigate the luminosity distribution of the FCOS objects. Can we confirm the soft transition 
Table 2. Table showing the mean velocities $\bar{v}$ and velocity dispersions $\sigma_{v}$ of all, only bright $(V<20$ mag) and only faint $(V>20$ mag) Fornax members. Samples considered are: the entire FCOS-sample (FCOS-1 and FCOS-2); the FCOS-sample corrected for the systematic velocity differences between FCOS-1 and FCOS-2 (see Sects. 3.1 and 4.1); and the shift corrected FCOS-sample + additional Fornax members from Dirsch et al. (2004) + the 2 UCDs not observed in the FCOS, taken from Karick et al. (2003). The column "Dif. Conf." gives the confidence limit in percent for the hypothesis of the bright and faint value being different, derived with a t-test for the means and with an F-test for the dispersions.

\begin{tabular}{l|cccc|cccc}
\hline \hline Samples & $\bar{v}_{\text {all }}$ & $\bar{v}_{\text {bright }}$ & $\bar{v}_{\text {faint }}$ & Dif. Conf. & $\sigma_{v, \text { all }}$ & $\sigma_{v, \text { bright }}$ & $\sigma_{v \text {,faint }}$ & Dif. Conf. \\
\hline FCOS & $1425 \pm 45$ & $1565 \pm 77$ & $1396 \pm 51$ & $85 \%$ & $326_{-32}^{+42}$ & $236_{-40}^{+86}$ & $335_{-36}^{+47}$ & $74 \%$ \\
FCOS shift-corrected (sc) & $1408 \pm 44$ & $1541 \pm 83$ & $1381 \pm 50$ & $83 \%$ & $322_{-32}^{+42}$ & $255_{-43}^{+93}$ & $328_{-35}^{+46}$ & $57 \%$ \\
FCOS sc + Dirsch + Karick & $1408 \pm 41$ & $1584 \pm 85$ & $1367 \pm 45$ & $96 \%$ & $326_{-30}^{+39}$ & $302_{-45}^{+96}$ & $320_{-32}^{+42}$ & $12 \%$ \\
\hline
\end{tabular}

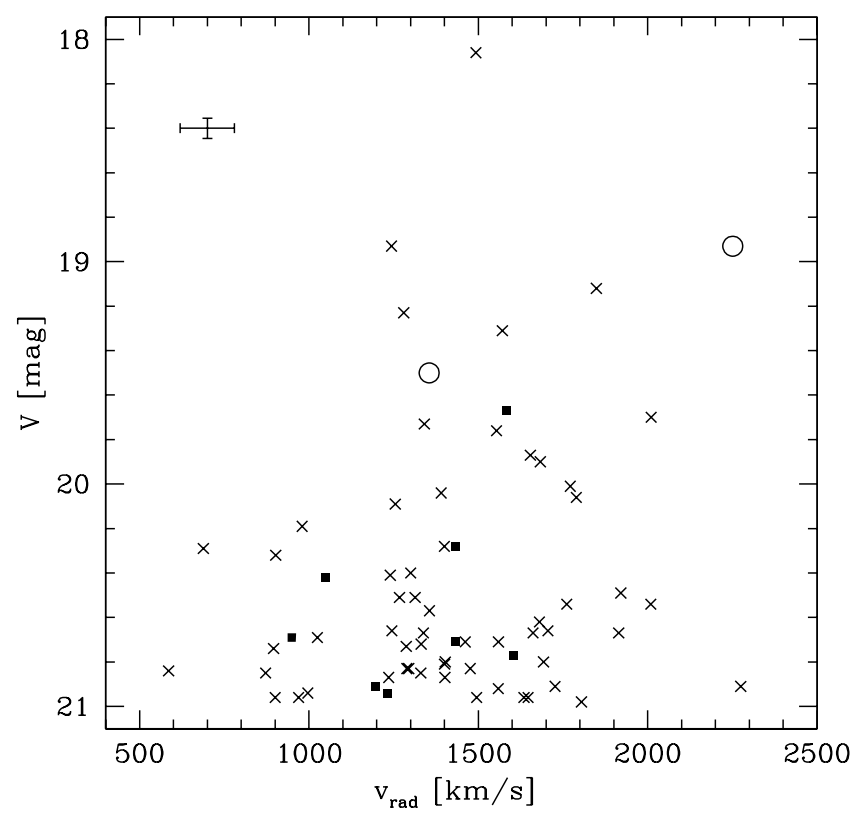

Fig. 6. Apparent $V$ magnitude plotted vs. radial velocity. Crosses: all FCOS Fornax members. Filled squares: Fornax members from Dirsch et al. (2004, private communications), not observed in the FCOS. Circles: the two UCDs not observed spectroscopically in the FCOS, with their radial velocities taken from Karick et al. (2003). The UCDs are those brighter than $V=19.5 \mathrm{mag}$. Typical errors are indicated by the error bars in the upper left.

in magnitude space between bright GCs and UCDs from Paper I, and is the sample sufficiently large to check for an overpopulation at the bright end?

\subsubsection{Survey completeness}

In order to investigate the magnitude distribution, it is necessary to calculate the overall survey completeness $C(r)$, which gives as a function of radius from NGC 1399 the probability than an object which satisfies our selection criteria is observed successfully ${ }^{2}$.

${ }^{2}$ The magnitude range brighter than $V=19.5$ mag is excluded from the analysis, as for this range the completeness is given by the FCSS to be $92.4 \%$ (Drinkwater, private communications), considered complete for our calculations.

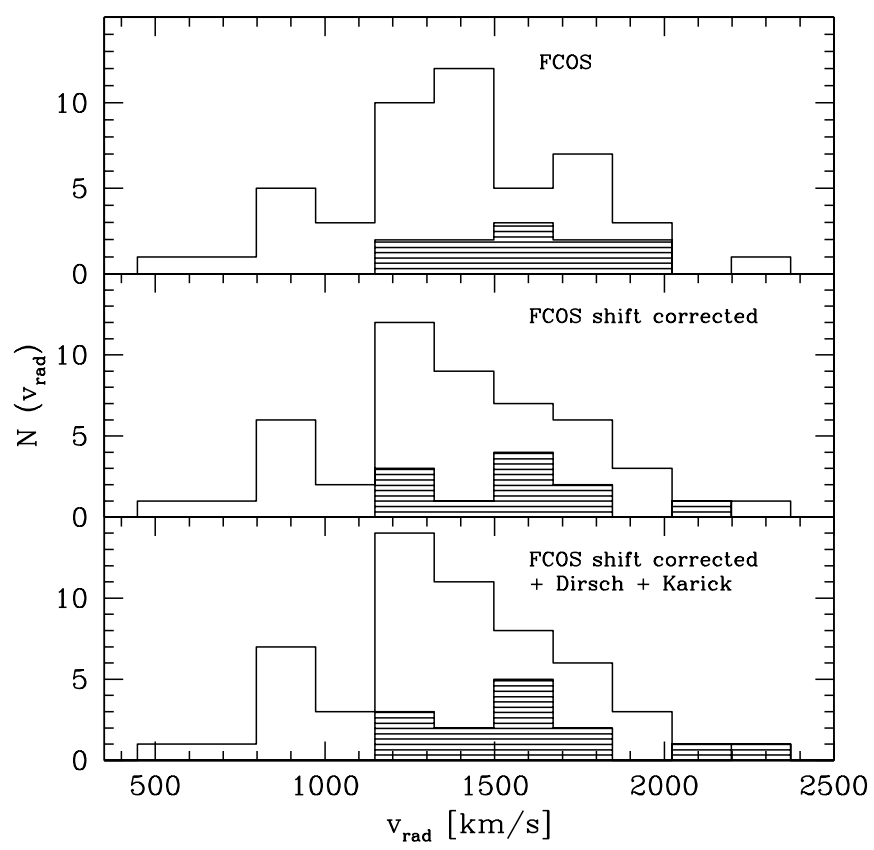

Fig. 7. Solid velocity histograms: Fornax members with $20<V<$ 21 mag. Shaded velocity histograms: Fornax members with $V<$ 20 mag. Top panel: FCOS objects. Middle panel: FCOS objects corrected for the systematic velocity shift between FCOS-1 and FCOS-2 (see Sects. 3.1 and 4.1). Bottom panel: FCOS shift corrected + additional Fornax members from Dirsch et al. (2004) + the 2 UCDs not observed in the FCOS, taken from Karick et al. (2003).

It holds:

$C(r)=C_{\mathrm{rv}}(r) \times C_{\mathrm{det}}(r) \times C_{\mathrm{geom}}(r)$

$C_{\mathrm{rv}}(r)$ is the number of objects with successfully determined radial velocity divided by the number of photometrically detected candidates. The global, radially integrated $C_{\mathrm{rv}}$ is $\frac{280}{462}=$ 0.606 , see Sect. 4. $C_{\text {det }}(r)$ is the photometric detection completeness of candidate objects. $C_{\text {geom }}(r)$ is the geometric incompleteness, caused by incomplete sky coverage of the fields from which candidates where selected. $C_{\text {det }}(r) \times C_{\text {geom }}(r)$ is obtained with artificial star experiments, where the number density per arcminute of input stars is constant for all investigated images, and the world coordinates of every input star are calculated. This allows to calculate the number density of recovered input stars as a function of distance to NGC 1399, yielding $C_{\text {det }}(r) \times C_{\text {geom }}(r)$. 


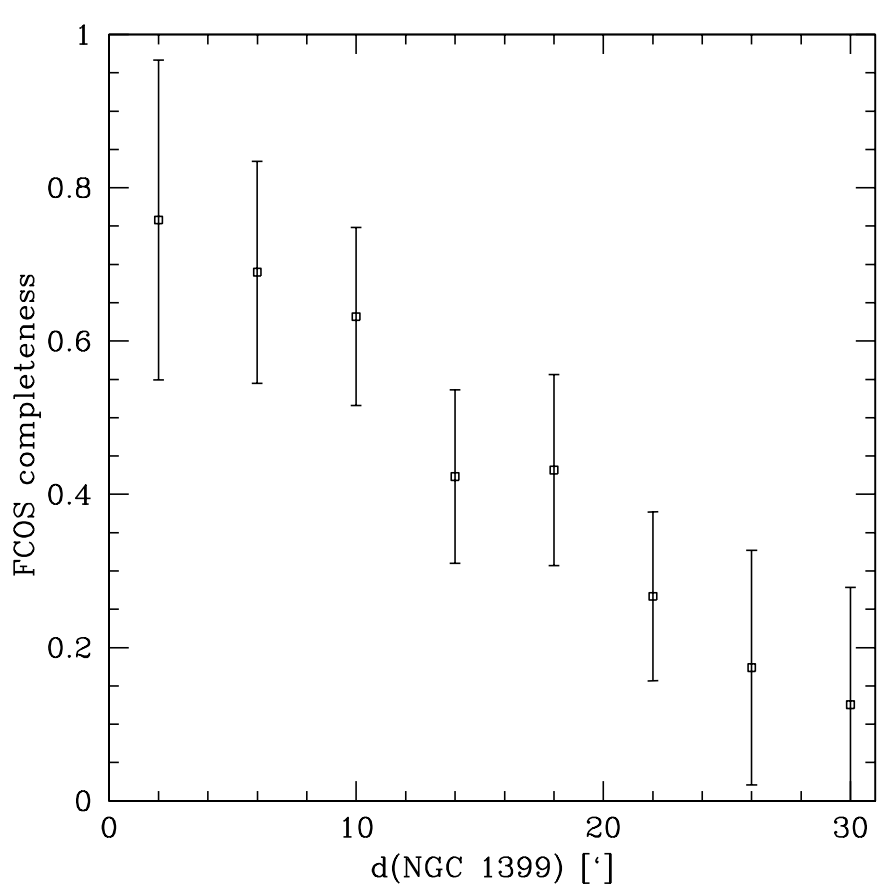

Fig. 8. Completeness of FCOS plotted vs. radial distance from NGC 1399. The completeness is defined as the ratio of the number of objects with successfully observed radial velocity and the number of existing candidates. For the latter value, both photometric and geometric completeness has to be calculated. See text for further details.

$C(r)$ is shown in Fig. 8. In the inner $10^{\prime}$, about $70 \%$ of existing candidates were successfully investigated, about $40 \%$ in the range between 10 and $20^{\prime}$. The completeness corrected total number of Fornax members is calculated by dividing the number of detected Fornax members in each radial bin by the completeness in the same bin. The total completeness $C_{0}$ for detecting Fornax members then becomes $61.5 \%$. $C_{0}$ is corrected for its magnitude dependence by subdividing the sample into bright $(19.5<V<20.25 \mathrm{mag})$ and faint $(20.25<V<21 \mathrm{mag})$ objects, and calculating $C_{0}$ separately for the two samples. $71 \%$ and $59 \%$ are obtained for bright and faint objects, respectively. At a given magnitude, $C_{0}$ is obtained by linear interpolation between the two values (see also Paper I).

\subsubsection{The form of the luminosity function at the bright end}

In Fig. 9, the incompleteness corrected luminosity distribution of FCOS Fornax members is shown. In addition, the luminosity distribution of the seven UCDs is given. The joined luminosity distribution of these two samples proves to be smooth and without any gaps until $V \simeq 18.5 \mathrm{mag}$. This confirms the result derived in Paper I. We therefore state that there is no separation in magnitude between the UCDs and the fainter compact objects in Fornax. Overplotted in Fig. 9 is a Gaussian luminosity function (LF) with the parameters $N_{\mathrm{GC}}=6450$, turn over magnitude $\mathrm{V}=24.0$ mag and width $\sigma=1.3 \mathrm{mag}$, as found for the GCS of NGC 1399 by Dirsch et al. (2003). The

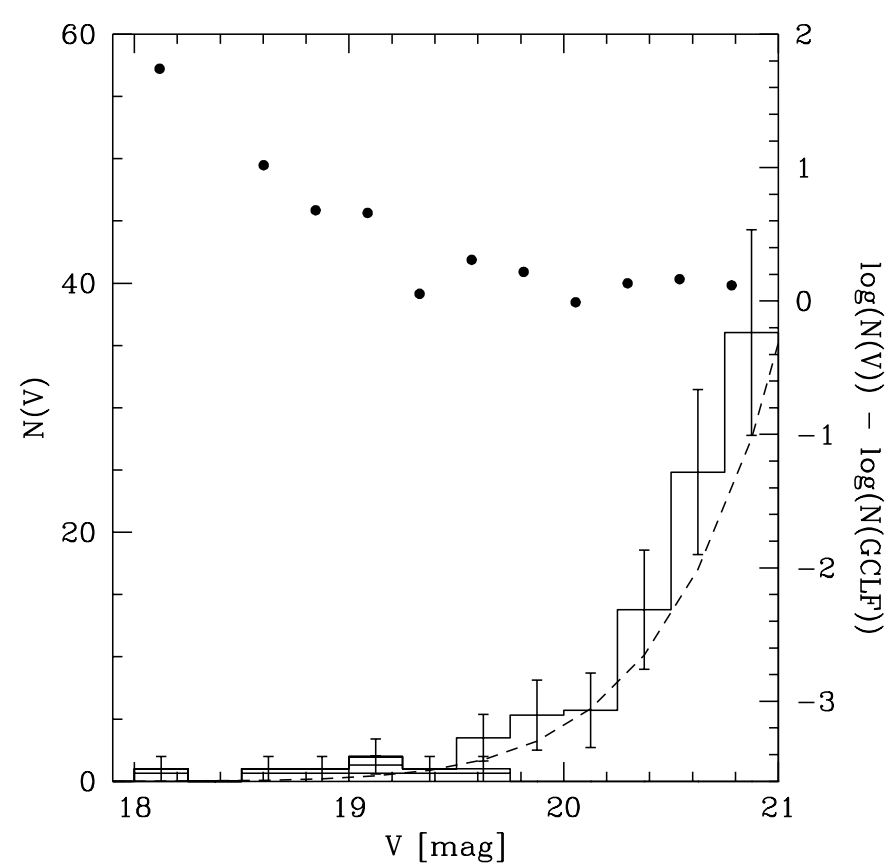

Fig. 9. Solid histogram: incompleteness corrected luminosity distribution $N(V)$ of the FCOS objects. Note that for the objects in the central field D1, only $C R$ photometry is available. Their $R$ magnitudes have been converted to the $V$-band via $V=R+0.5 \mathrm{mag}$. See Sect. 2.2 for further details. Shaded histogram: luminosity distribution of the seven UCDs. Dashed line: Gaussian LF for the GCLF of NGC 1399, taken from Dirsch et al. (2003). The parameters are: $N_{\mathrm{GC}}=6450$, turn over magnitude $V=24.0$ mag, width $\sigma=1.3$ mag. Filled circles: Logarithm of the ratio $\frac{N(V)}{N(\mathrm{GCLF})}$.

paper of Dirsch et al. presents the most complete photometric survey of NGC 1399's globular cluster system, yet. The LF fit to their results is a good approximation of the real magnitude distribution in the magnitude range observed by us, although for very bright magnitudes it slightly underestimates the number counts. As the real magnitude distribution is smooth and without any significant peaks, we characterize the shape of its bright end by overplotting in Fig. 9 the log-ratio of the number of objects found by us and the number expected from the LF by Dirsch et al. This ratio is close to unity for magnitudes fainter than $V \simeq 20 \mathrm{mag}$, but rises strongly for brighter magnitudes. The magnitude where this ratio change occurs agrees roughly with the limit of $V=20$ mag between the objects with higher and lower mean velocity found in Sect. 4.1. For the entire range $V<20 \mathrm{mag}$, the incompleteness corrected overpopulation with respect to the Gaussian luminosity function from Dirsch et al. is $120 \% \pm 65 \%$, corresponding to $8 \pm 4$ objects. This is only slightly lower than the estimated total number of 14 UCDs as predicted by Bekki et al. (2003).

Note, however, that the overpopulation found here is with respect to the extrapolation of the GCLF fitted at fainter magnitudes, which is not sufficient to prove that there really is one. See Sect. 5 for further discussion. 


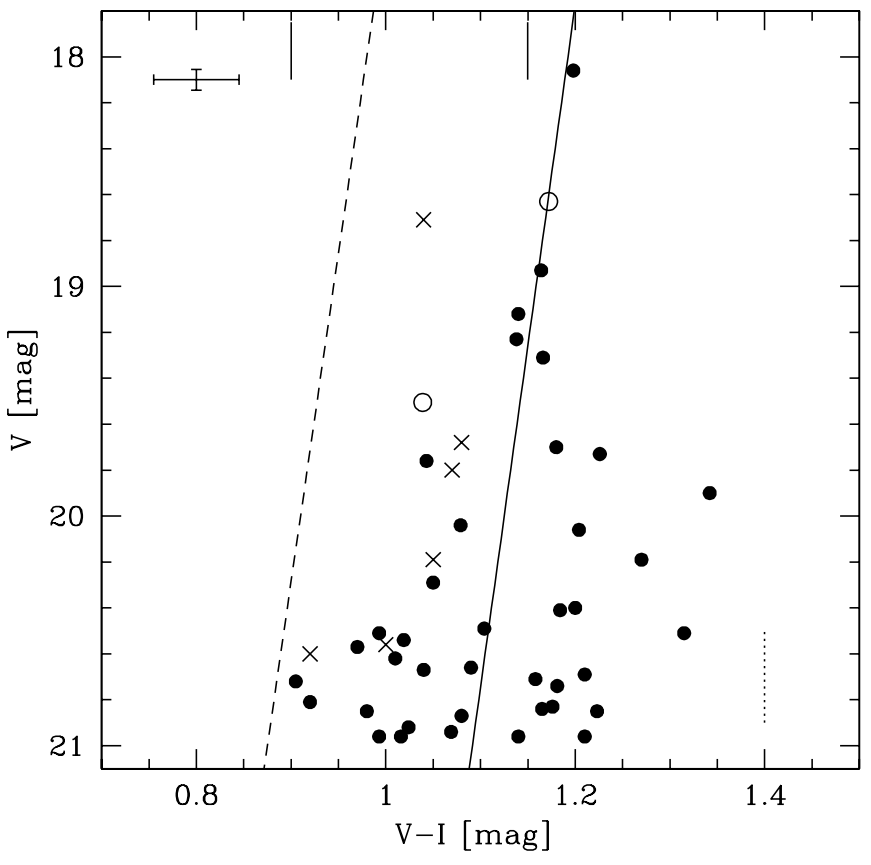

Fig. 10. CMD of the compact Fornax members. Filled circles are the FCOS objects with VI photometry available (from Hilker et al. 2003). The open circles are the 2 UCDs not observed in the FCOS. Crosses mark VI photometry of Fornax and Virgo dE,N nuclei, taken from Lotz et al. (2001). The solid line is a linear fit to the circles applying a $2 \sigma$ rejection algorithm. When not applying the $2 \sigma$ rejection, the resulting slope increases by about $30 \%$ and the overall fit is about 0.02 mag redder than the colours of the brightest objects. The dashed line is the CM relation found for Fornax dEs by Hilker et al. (2003). The vertical ticks at $(V-I)=0.90$ and 1.15 mag indicate the location of the blue and red peak of NGC 1399's bimodal GC colour distribution from Gebhardt \& Kissler-Patig (1999). The dotted vertical tick at $(V-I)=1.40$ mag indicates the red colour limit for FCOS-2 candidate selection. For FCOS-1, the limit had been 1.50 mag (see also Sect. 2.1).

\subsection{Colour-magnitude diagram}

The mean colour of GC systems in early type galaxies is between $(V-I)=1.00$ and 1.05 (e.g. Kundu \& Whitmore 2001a,b). The blue and red peaks of the frequently detected bimodal colour distribution generally lie at about 0.95 and $1.18 \mathrm{mag}$, respectively (Kundu \& Whitmore 2001a). No $\mathrm{CM}$ relation in the sense that within one globular cluster system, the GC colour becomes redder with brighter GC magnitude, has been reported for any GC system. This is in contrast to dEs, for which a tight CM relation exists (e.g. Hilker et al. 2003).

In the case of NGC 1399, Gebhardt \& Kissler-Patig (1999) find from HST WFPC2 archive data a bimodal colour distribution with peaks at $(V-I) \simeq 0.9$ and $1.15 \mathrm{mag}$, respectively. Dirsch et al. (2003) find with VLT FORS1-photometry a unimodal colour distribution for bright GCs, with a mean between 1.05 and $1.1 \mathrm{mag}$.

Figure 10 shows a CM diagram in $V$ and $(V-I)$ of the FCOS objects, including the UCDs. VI photometry of dE,N nuclei derived by Lotz et al. (2001) for Fornax and Virgo cluster dE,Ns is shown as well.
The CMD has three main features:

1. there is a CM relation for the FCOS objects with a very similar slope to the relation found for Fornax dEs by Hilker et al. (2003), but shifted about 0.20 mag redwards. The slope $\frac{\delta(V-I)}{\delta(V)}$ found for the FCOS objects is $-0.034 \pm 0.011$, significant at $3 \sigma$. This slope was fitted using a $2 \sigma$ clipping algorithm. When not doing any clipping, the slope rises to $-0.044 \pm 0.011$, but provides a poorer fit to the brightest data points. For the Fornax dEs the slope is -0.036 . When excluding the brightest 3 data points from the sigma clipping fit, the resulting slope is $-0.038 \pm 0.015$. This shows that the existence of the slope is not only defined by the brightest data points. When excluding instead the faint compact objects $(V>20 \mathrm{mag})$ from the same fit, the slope becomes $-0.041 \pm 0.027$, i.e. still consistent with the overall slope but only half as significant. Finally, excluding from the data points used for the last fit also the brightest data point, changes the slope only slightly to $-0.035 \pm 0.039$. All these fits to the different restricted data samples indicate that the existence of the slope stands on firm ground. For the faint compact objects, the scatter in $(V-I)$ is about $0.10 \mathrm{mag}$, too large to reliably measure a slope. The red colour limit of $(V-I)=1.40 \mathrm{mag}$ for FCOS-candidates has no effect on the slope of the relation, as the colour space $1.20<(V-I)<1.40 \mathrm{mag}$ is only very sparsely populated. Note that already Karick et al. (2003) have found a colouroffset between dwarf galaxies and UCDs in Fornax, but their colour precision and sample size was not sufficient to detect a possible CM-relation for the UCDs;

2. the colours of dE,N nuclei from Lotz et al. (2001) lie between dEs and FCOS objects, shifted about 0.1 mag redwards with respect to dEs and about 0.1 mag bluewards of FCOS objects;

3. the mean colour of bright compact objects $((V-I)=1.16 \pm$ 0.02 ) is redder than the average mean for GC systems, consistent with the metal-rich peak of the bimodal colour distribution. Their overall colour range is $1.0<(V-I)<1.3$. The mean colour of the faint compact objects $((V-I)=$ $1.10 \pm 0.02)$ and their colour range of $0.9<(V-I)<1.3 \mathrm{mag}$ is slightly more consistent with the overall mean colours of GCs.

The existence of a CM relation for the bright compact objects as found here is a property that has not been reported for any GCS, yet. In Sect. 5 this finding will be further discussed.

\subsection{Radial density distribution}

Figure 11 shows the cumulative radial distribution of bright and faint compact objects plus the radial distribution of NGC 1399's GC system as investigated by Dirsch et al. (2004). Note that Dirsch et al. find that the GCs contained in the blue peak of NGC 1399's bimodal colour distribution have a significantly higher velocity dispersion, mean velocity and more extended spatial distribution than the GCs in the red peak. Therefore we compare our values with the Dirsch et al. sample restricted to the same colour range as ours, which is $(V-I)>0.9$ (see Fig. 10), corresponding to $(C-R)>1.4$ 

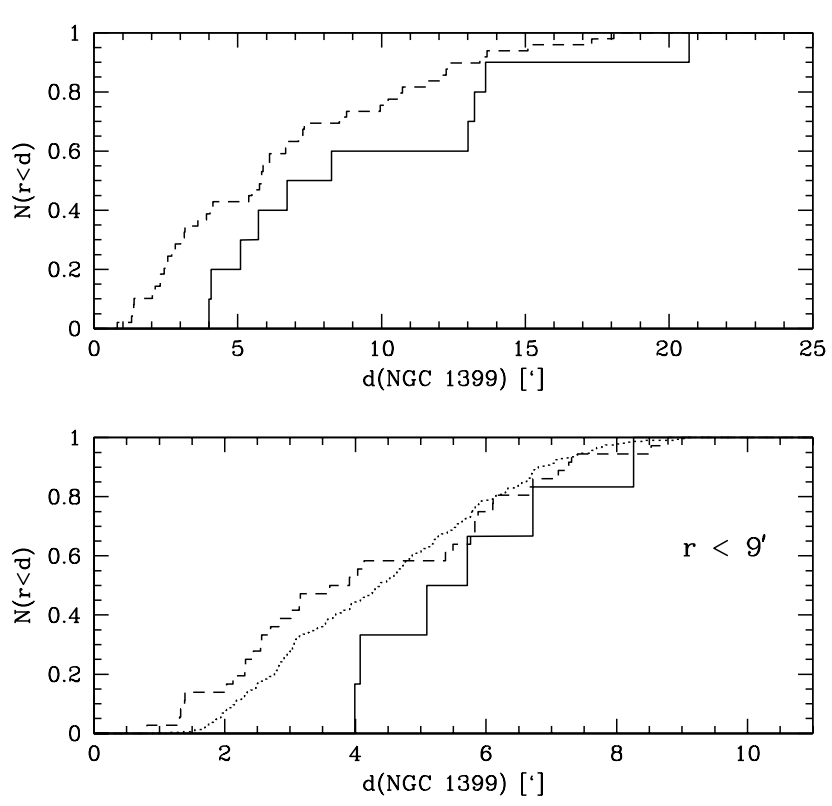

Fig. 11. Top panel: cumulative radial distributions of FCOS objects. Solid histogram: bright compact objects $(V<20 \mathrm{mag})$. Dashed histogram: faint compact objects $(V>20 \mathrm{mag})$. Bottom panel: comparison between cumulative radial distribution of FCOS objects and GCs from Dirsch et al. (2004), restricted to $9^{\prime}$, as this is the extension of the Dirsch sample. Solid and dashed histogram as in top panel. Dotted histogram: GCs from Dirsch et al. with $(C-R)>1.4$ (see text).

(Worthey et al. 1994). The following conclusions are extracted from Fig. 11:

1. the bright compact objects are slightly less concentrated towards NGC 1399 than the faint ones. Applying a KS-test shows that the sample of bright objects is drawn from a different distribution than the faint objects at $88 \%$ probability;

2. the radial distribution of NGC 1399 's GCS within 9' agrees slightly better with the faint than the bright compact objects. Applying a KS-test shows that the faint compact objects are drawn from a different distribution than the entire GCS at $71 \%$ confidence, while the bright ones are inconsistent at the $86 \%$ confidence level.

These findings agree with the separation between bright and faint compact objects in radial velocity (Sect. 4.1) and hence add further credibility to that distinction.

\section{Discussion}

In the following, we will discuss to what extent our findings provide support for the hypothesis that the UCDs and possibly other bright stellar clusters around NGC 1399 do not belong to NGC 1399's GCS but rather are remnants of threshed dE,Ns. Also, we consider the possibility of stellar super-clusters created in past merging events.

\subsection{Explanation for dynamically distinct subgroups}

A difference in mean radial velocity has been found between the bright $(V<20 \mathrm{mag})$ and faint $(V>20 \mathrm{mag})$ compact objects in Sect. 4.1. See Table 2 for exact numbers. We compare these results with values published in the literature for the Fornax cluster.

Dirsch et al. (2004, and private communications) obtain a mean velocity of $1440 \pm 15 \mathrm{~km} \mathrm{~s}^{-1}$ with a dispersion of $325 \mathrm{~km} \mathrm{~s}^{-1} \pm 11 \mathrm{~km} \mathrm{~s}^{-1}$ for a sample of almost $500 \mathrm{GCs}$ brighter than $V=23 \mathrm{mag}$, when restricting their sample to radial velocities higher than $550 \mathrm{~km} \mathrm{~s}^{-1}$, as done in this paper. These values are in agreement with our results for the entire FCOS sample, see Table 2. The same table shows that the mean velocity of the Dirsch sample is slightly more consistent with the faint than the bright compact objects. Due to the different spatial and velocity distribution found by Dirsch et al. for the red and blue GC sample, it seems however more appropriate to compare our velocity values with their colour restricted sample, i.e. for $(C-R)>1.4$, as already pointed out in see Sect. 4.4.

The mean velocity of Dirsch et al.'s colour restricted sample is $1422 \pm 17 \mathrm{~km} \mathrm{~s}^{-1}$ with a dispersion of $309 \pm 13 \mathrm{~km} \mathrm{~s}^{-1}$. The velocity dispersion agrees well with our entire sample and the sub-samples. A t-test shows that the mean velocity of Dirsch's sample is different at $79 \%$ confidence $(1.2 \sigma)$ from the faint compact objects in the shift corrected enlarged sample and different at $93.5 \%$ confidence $(1.9 \sigma)$ from the bright ones in the same sample. For the shift-corrected not enlarged sample the confidence limits for different means are $60 \%$ for the faint objects and $77 \%$ for the bright ones.

Thus, our findings show that the mean velocity of faint compact objects is more consistent with NGC 1399's GCS than that of the bright ones.

In that context it is natural to ask how our values, especially for the bright compact objects, compare with what is found for the dwarf galaxy population of Fornax, because the threshing scenario predicts dwarf galaxies as the progenitor objects of UCDs.

The most recent and complete spectroscopic observations of Fornax cluster galaxies come from Drinkwater et al. (2001a,b). Separating their Fornax main cluster sample into dwarfs and giants with $b_{j}=15$ mag as the limiting magnitude, one obtains a mean radial velocity of $1511 \pm 56 \mathrm{~km} \mathrm{~s}^{-1}$ and a dispersion of $406 \pm 36 \mathrm{~km} \mathrm{~s}^{-1}$ for the dwarf galaxies, while the giants have $1426 \pm 52 \mathrm{~km} \mathrm{~s}^{-1}$ and $314 \pm 33 \mathrm{~km} \mathrm{~s}^{-1}$. The latter values are consistent with the faint compact objects and Dirsch et al.'s GCs. Drinkwater et al. suggest that the difference in dispersion is due to the fact that the Fornax dwarf galaxies are not yet virialized, but still falling into the cluster. This might as well explain the small offset in radial velocity between dwarfs and giants, assuming that the dwarfs have an anisotropic infall.

Comparing the different mean velocities for Fornax dwarf and giant galaxies from Drinkwater et al. with our results using a t-test shows that the mean velocity of the bright compact objects is consistent with that of the dwarf galaxies, yielding only $45 \%$ confidence for the hypothesis of a different mean. It is inconsistent at $95 \%$ confidence with that of the giants. For the comparison of faint compact objects to dwarfs and giants, the confidence levels for a different mean are $90 \%$ and $60 \%$, respectively. 
This supports the hypothesis that UCDs and bright compact objects $(V<20 \mathrm{mag})$ originate mainly from dwarf galaxies, while the faint compact objects $(V>20 \mathrm{mag})$ are mainly genuine GCs.

However, there is a difference of $104 \pm 102 \mathrm{~km} \mathrm{~s}^{-1}$ between the velocity dispersions of bright compact objects in the shift corrected enlarged sample and Fornax dwarf galaxies. Although this is only marginally significant, a possible reason for this difference is given by Bekki et al. (2001, 2003 and private communications). They find from their simulations that both highly eccentric orbits $\left(e_{\mathrm{p}}>0.7\right)$ and smaller pericenter distance are required for the transformation from dE,Ns to UCDs. These restrictions mean that the UCDs have a smaller velocity dispersion than the non-threshed $\mathrm{dEs}$ or $\mathrm{dE}$,Ns. Thus the dispersion of UCDs around the mean cluster velocity should be smaller than that of average dEs or dE,Ns, which qualitatively explains our findings. Note that the dispersion of the bright compact objects around the mean velocity of the Fornax giants $\left(1426 \mathrm{~km} \mathrm{~s}^{-1}\right)$ is $335 \mathrm{~km} \mathrm{~s}^{-1}$ for the shift corrected enlarged sample, i.e. still somewhat smaller than that of average dwarf galaxies.

An interesting, speculative consideration is: if the threshing affects a $\mathrm{dE}$ population with smaller velocity dispersion than the mean cluster dispersion, then the velocity standard deviation of this population is lower and therefore the velocity values are concentrated stronger around the mean than for the rest of the population. This means that by excluding the threshed population, which has a smaller velocity dispersion, from the entire sample of dEs, the velocity distribution of the remaining non-threshed dEs should broaden. To estimate the magnitude of this effect, we add to the velocity distribution of dwarf galaxies from Drinkwater et al. (2001a,b) twice the velocities of bright compact objects from this paper in order to also take into account entirely disrupted dEs. The resulting velocity dispersion is $370 \mathrm{~km} \mathrm{~s}^{-1}$, compared to $406 \mathrm{~km} \mathrm{~s}^{-1}$ for the original sample. This speculative calculation shows that the threshing might be partially responsible for the higher velocity dispersion of Fornax dwarf galaxies. Further discussion of this is, however, beyond the scope of this paper.

An additional process to create UCDs has been brought forward by Fellhauer \& Kroupa (2002), who show that massive stellar super-clusters formed in violent merging events can after few Gyrs resemble the properties of UCDs. In the case of the very dense Fornax cluster, the probability of a major merging occurred in the past is very high. The GCS of NGC 1399 has a pronounced bimodality (Gebhardt \& Kissler-Patig 1999; Dirsch et al. 2003), suggesting that at least two separated star forming events have occurred. If the bright compact objects were such super-clusters, one might expect to find a trace of the merging event like a different velocity than the Fornax mean, alignment along a preferred axis or association with major Fornax galaxies. While a different (higher) velocity has been found, Fig. 4 shows that there is no alignment for the bright compact objects, nor are they distributed especially close to a Fornax cluster galaxy other than the central galaxy NGC 1399. However, this does not rule out the super-cluster scenario, as after several Gyrs the traces of the merger event, be it in velocity or 2D-space, become smeared out. In fact, detailled simulations regarding this scenario for the Fornax cluster case would be needed to better assess its validity.

\subsection{Colour magnitude relation for the FCOS Fornax members}

It has been shown in Sect. 4.3 that there is a CM relation for the FCOS Fornax members with a slope agreeing with what is found for dEs. This relation is shifted about 0.2 mag redwards with respect to the $\mathrm{dE}$ relation. The colours of $\mathrm{dE}, \mathrm{N}$ nuclei lie between those of dEs and FCOS objects. While the bright compact objects $(V<20$ mag) follow a slope consistent with the overall one, the faint compact objects $(V>20 \mathrm{mag})$ scatter too much in $(V-I)$ in order to reliably confirm or discard the existence of a slope in that magnitude range. With a Gedankenexperiment it can be estimated, where the CM relation for threshed nuclei should be situated with respect to that of the dEs:

One assumes that the nucleus has the same colour as the entire $\mathrm{dE}, \mathrm{N}$ and that in the course of the threshing, the stellar population of a $\mathrm{dE}, \mathrm{N}$ and especially that of its nucleus is not altered. Hence, only the dE,N mass and thereby its luminosity are decreased in the threshing process until the naked nucleus is left over and an UCD is created. In a CMD this means that in the course of the transformation from dE,N to UCD, a galaxy moves in the $y$-axis (magnitude) by the amount corresponding to its luminosity-loss. The CM relation defined by the newly formed UCDs would then be shifted redwards by its slope multiplied with the magnitude difference between $\mathrm{dE}, \mathrm{N}$ and $\mathrm{UCD}$, assuming that this difference does not depend strongly on the dE,N magnitude. Bekki et al. (2001, 2003) calculate for their fiducial model that in the threshing process, the magnitude difference between the original $\mathrm{dE}, \mathrm{N}$ and the final UCD is $4.1 \mathrm{mag}$. Such a magnitude difference corresponds to a redward shift of the CM relation of about $0.15 \mathrm{mag}$, as the slope of the $\mathrm{dE} C M$ relation is about -0.036 . This brings the shifted CM relation of dEs very close (about $0.07 \mathrm{mag}$ ) to the one found in this paper for the FCOS objects. The data of Lotz et al. (2001) suggest that the mean magnitude difference between a dE,N and its nucleus is about $5 \mathrm{mag}, 1 \mathrm{mag}$ larger than the difference given by Bekki et al. for their fiducial model. In that case, the shift of the CM relation between dE,Ns and stripped nuclei would be about $0.18 \mathrm{mag}$ and bring to match both relations within the colour scatter of our data.

Thus, with a simple but plausible assumption the threshing scenario predicts a CM relation for threshed nuclei with the same slope and the same zero point (to within $0.05 \mathrm{mag}$ ) as observed for the FCOS objects.

However, the condition that the $\mathrm{dE}, \mathrm{N}$ nuclei have the same colour as the entire galaxy, is probably an oversimplification. HST photometry of Virgo and Fornax dEs and dE,N nuclei (Stiavelli et al. 2001; Lotz et al. 2001) shows that the nuclei are on average about 0.07 mag bluer than the underlying galaxy light. If the progenitor $\mathrm{dE}, \mathrm{Ns}$ of UCDs would have the same properties as the dE,Ns and nuclei investigated by Lotz et al., the UCDs would be expected to be about $0.10 \mathrm{mag}$ bluer than they actually are. Indeed, the nuclei of Lotz et al. are 
between 0.05 and 0.10 mag bluer than the bright compact objects, including the UCDs (see Fig. 10).

This discrepancy could be explained, if the already stripped nuclei tended to contain older populations than the dE,Ns and their nuclei as investigated by Lotz et al. (2001). This is a plausible assumption for two reasons:

(1) the tidal threshing strongly reduces the gravitational potential well of the threshed galaxy both in its center and its outskirts, and thus less gas than compared to nonthreshed $\mathrm{dE}$,Ns can be retained for possible star forming bursts;

(2) about 5 Gyrs is a minimum age for UCDs (Bekki et al. 2003), as this is about the time-frame needed for complete stripping.

To get an idea of the age differences needed to explain the observed colour difference between UCDs and dE,N nuclei, stellar population models by Worthey (1994) are used. We find that for a population of $\mathrm{Fe} / \mathrm{H}=-0.225$ and age $5 \mathrm{Gyrs}$, $(V-I)=1.08$. For the same metallicity but age 10 Gyrs, we obtain $(V-I)=1.18$. In other words, an age difference of the time needed to transform a dE,N into a UCD can explain the colour difference between the bright compact objects and the dE,N nuclei.

An alternative explanation for our finding could be a globular cluster CM relation in the sense that within one GCS, brighter GCs become increasingly redder. To our knowledge the relation found by us would then be the first one reported for a GCS, yet. The idea is that brighter and more massive clusters, e.g. the stellar super-clusters created in mergers (Fellhauer \& Kroupa 2002, become increasingly more self-enriched with growing mass (e.g. Platais et al. 2003; Meylan et al. 2001). Very massive GCs would on average have redder colours than less massive ones (of the same GCS), just as found by us. The merger event could also be responsible for the dynamical differences found by us between bright and faint compact objects. However, the idea of a mass-metallicity relation has been questioned by Parmentier \& Gilmore (2001), who have shown that the old galactic halo globular clusters seem to follow a reverse magnitude-metallicity relation, with less massive clusters having higher metallicities. They claim that this anticorrelation is as predicted by self-enrichment models.

Note that a CM relation for massive GCs as it might have been found by us, cannot be backed up with earlier findings of such a relation but stands on its own. In contrast, the CM relation for dwarf galaxies, from which the UCDs originate according to the threshing scenario, is a well established fact (Hilker et al. 2003).

We therefore believe that based on our present knowledge, the $\mathrm{CM}$ relation found by us is more readily explained with the threshing scenario and a galactic origin of the UCDs rather than with massive GCs following a CM relation. The fact that the slope is well defined for $V<20$ mag is consistent with the proposed magnitude separation of $V=20$ mag between threshed nuclei and normal GCs, as proposed in the previous section. There can also be fainter UCDs with $V>20$ mag or GCs with $V<20 \mathrm{mag}$, but our findings suggest that in the respective magnitude regimes they are in the minority.

\subsection{Overpopulation at the bright end}

In Sect. 4.2.2 it was shown that the number of bright compact objects is more than two times higher than expected from the GCLF of Dirsch et al. (2003), which is the best determined GCLF of NGC 1399 up to now. For $V<20$ mag, there are $8 \pm 4$ more objects than expected from integrating the GCLF of Dirsch et al. This overpopulation is consistent with the threshing scenario as a source of bright compact objects which add to the LF of very bright GCs. Bekki et al. (2003) have shown that the total number of UCDs in the Fornax cluster should be about $10-15$, which agrees roughly with our finding. Apart from threshed dE,Ns, it is clear that also the superclusters proposed by Fellhauer \& Kroupa (2002) could add to the number counts at bright magnitudes.

However, the overpopulation found by us is only with respect to the extrapolation of the LF fit by Dirsch et al. to their data at fainter magnitudes (the turn-over magnitude is at about $V=24.0 \mathrm{mag}$ ). The contamination by background galaxies or foreground stars for $V<20$ in the FCOS is about $90 \%$, illustrating that Dirsch et al. could not use this part of the magnitude distribution for fitting their LF.

Therefore, only if the entire GCLF of NGC 1399 would be described well by Dirsch et al.'s fit also at brighter magnitudes, the luminosity distribution of FCOS members found by us supports a slight overpopulation for $V<20 \mathrm{mag}$, caused either by threshed dE,Ns or stellar super-clusters.

\section{Summary and conclusions}

In this paper, we have presented the second part FCOS-2 of the Fornax Compact Object Survey FCOS and investigated the merged data sets of FCOS-1 (Paper I) and FCOS-2. The aim of FCOS was to obtain more clues on the nature of the socalled "Ultra Compact Dwarf Galaxies" (UCDs), as detected in the central Fornax cluster and described by Drinkwater et al. (2003). 280 objects in the magnitude space $18<V<21 \mathrm{mag}$, covering both UCDs and bright globular clusters in Fornax, were observed spectroscopically to determine their cluster membership. Their distribution in radial velocity, colour, magnitude and space was investigated. The following results have been obtained:

1. 54 new compact Fornax members in the magnitude range $19.7<V<21 \mathrm{mag}$ were detected. In addition, 5 of the 7 previously discovered UCDs were observed, covering a magnitude range of $18<V<19.5 \mathrm{mag}$.

2. The radial velocity distribution is different for bright $(V<$ $20 \mathrm{mag}$ ) and faint ( $V>20 \mathrm{mag})$ compact objects. At the $96 \%(2.1 \sigma)$ confidence level, the bright ones have a higher mean velocity than the faint ones. The result for the faint compact objects is more consistent with existing studies on the GCS of NGC 1399 than for the bright ones. The bright compact objects have a mean velocity consistent with the mean dwarf galaxy velocity in the Fornax cluster, but a lower velocity dispersion than the dwarf galaxies. This smaller velocity dispersion is explained qualitatively within the threshing scenario by Bekki et al. (2003). Our result is therefore consistent with the threshing scenario as a source of bright compact objects and suggests that UCDs created 
by threshing dE,Ns may extend down to $V \simeq 20$ mag. Our findings are also consistent with the super-cluster scenario as a source of UCDs (Fellhauer \& Kroupa 2002), but the range of observed properties consistent with this scenario is larger than in the threshing scenario.

3. The FCOS Fornax members follow a CM relation with a very similar slope to that of dEs, but shifted about 0.2 mag redwards. This shift is explained well by the threshing scenario, assuming that the threshed $\mathrm{dE}, \mathrm{N}$ keeps its colour in the course of the tidal interaction and only loses luminosity. The bluer colours of dE,N nuclei compared to bright FCOS objects are explained by assuming an age difference between the two populations of the time needed to transform a $\mathrm{dE}, \mathrm{N}$ into a UCD. An alternative explanation for the CM relation is that the bright FCOS objects are super-massive GCs created in a merger which follow a mass-metallicity relation in the sense that mass increases with metallicity. This would be the first time that such a relation is detected for a GCS.

4. The magnitude distribution of FCOS Fornax members shows a fluent transition between UCDs and GCs and a slight overpopulation of $8 \pm 4$ objects for $V<20 \mathrm{mag}$ with respect to the extrapolation of the GCLF by Dirsch et al. (2003), which matches roughly the predicted number of UCDs in the threshing scenario. This finding is also consistent with the super-cluster-scenario as a source of additional compact objects in the given magnitude regime. However, it is not sufficient to solidly support either scenario because the reference GCLF was determined at fainter magnitudes than investigated here.

5. The bright FCOS objects have a more extended spatial distribution than the faint ones at $88 \%$ confidence and have a distribution inconsistent with the GCS of NGC 1399 at $86 \%$ confidence. The distribution of faint FCOS objects is more consistent with NGC 1399's GCS.

Our results are consistent with a substantial fraction of bright compact objects ( $V<20 \mathrm{mag}, M_{V}<-11.4 \mathrm{mag}$ ) being nuclei of threshed $\mathrm{dE}, \mathrm{Ns}$, whereas the fainter ones are mainly normal GCs of NGC 1399's very rich GCS. The existence of stellar super-clusters following a CM relation as an alternative source of bright compact objects is also consistent with our data.

Defining UCDs as extremely bright star clusters distinct from genuine globular clusters, our findings show that in Fornax the UCDs extend to at least $M_{V}=-11.4(V=20) \mathrm{mag}$, about 0.5 mag deeper than the 7 UCDs found by Drinkwater et al. (2000a). The UCDs populate the colour range $1.0<$ $(V-I)<1.3$. For $M_{V}>-11.4(V>20) \mathrm{mag}$, there can also be UCDs with possibly bluer colours than given above which mix up with genuine GCs.

Doing a survey similar to FCOS in Virgo or other massive nearby clusters where higher numbers of UCDs are expected (Bekki et al. 2003) would be the next step to compare properties of UCDs in different environments.

Acknowledgements. We thank the referee Dr. Michael Drinkwater for his comments which improved the paper. The authors are very grateful to Dr. Boris Dirsch for providing us with his data and for helpful discussions. We thank Dr. Kenji Bekki for providing us with information about the dynamical implications of the threshing scenario. S.M. was supported by DAAD Ph.D. grant Kennziffer D/01/35298 and DFG Projekt Nr. HI 855/1-1. LI acknowledges support from proyecto FONDAP \# 15010003.

\section{References}

Bekki, K., Couch, W. J., \& Drinkwater, M. J. 2001, ApJ, 552, L105

Bekki, K., Couch, W. J., Drinkwater, M. J., \& Shioya, Y. 2003, MNRAS, 344, 399

Bertin, E., \& Arnouts, S. 1996, A\&AS, 117, 393

Dirsch, B., Richtler, T., Geisler, D., et al. 2003, AJ, 125, 1908

Dirsch, B., Richtler, T., Geisler, D., et al. 2004, AJ, in press

Djorgovski, S. G., Gal, R. R., \& McCarthy, J. K. 1997, ApJ, 474, L19

Drinkwater, M. J., Jones, J. B., Gregg, M. D., \& Phillipps, S. 2000a, PASA, 17, 227

Drinkwater, M. J., Phillipps, S., Jones, J. B., et al. 2000b, A\&A, 355, 900

Drinkwater, M. J., Gregg, M. D., Holman, B. A., \& Brown, M. J. I. 2001a, MNRAS, 326, 1076

Drinkwater, M. J., Gregg, M. D., \& Colless, M. 2001b, ApJ, 548, L139

Drinkwater, M. J., Gregg, M. D., Hilker, M., et al. 2003, Nature, 423, 519

Faber, S. M., \& Jackson, R. E. 1976, ApJ, 204, 668

Fellhauer, M., \& Kroupa, P. 2002, MNRAS, 330, 642

Ferguson, H. C., \& Sandage, A. 1988, AJ, 96, 1520

Ferguson, H. C., \& Sandage, A. 1989, ApJ, 346, 53

Ferrarese, L., Ford, H. C., \& Huchra, J. 2000, ApJS, 128, 431

Gebhardt, K., \& Kissler-Patig, M. 1999, AJ, 118, 1526

Graham, A. W. 2002, ApJ, 568, L13

Hilker, M., Infante, L., \& Richtler, T. 1999, A\&AS, 138, 55

Hilker, M., \& Richtler, T. 2000, A\&A, 362, 895

Hilker, M., Mieske, S., \& Infante, L. 2003, A\&A, 397, L9

Hilker, M., Mieske, S., \& Infante, L. 2004, in prep.

Karick, A., Drinkwater, M. J., \& Gregg, M. D. 2003, MNRAS, 344, 188

Kroupa, P. 1998, MNRAS, 300, 200

Kundu, A., \& Whitmore, B. C. 2001a, AJ, 121, 2950

Kundu, A., \& Whitmore, B. C. 2001b, AJ, 122, 1251

Lotz, J. M., Telford, R., Ferguson, H. C., et al. 2001, ApJ, 552, 572

Maraston, C., Bastian, N., Saglia, R. P., et al. 2004, A\&A, 416, 467

Meylan, G. 1987, A\&A, 184, 144

Meylan, G., Mayor, M., Duquennoy, A., \& Dubath, P. 1994, BAAS, 26, 956

Meylan, G., Mayor, M., Duquennoy, A., \& Dubath, P. 1995, A\&A, 303, 761

Meylan, G., Sarajedini, A., \& Jablonka, P. 2001, AJ, 122, 830

Mieske, S., Hilker, M., \& Infante, L. 2002, A\&A, 383, 832 (Paper I)

Parmentier, G., \& Gilmore, G. 2001, A\&A, 378, 97

Platais, I., Wyse, R. F. G., Hebb, L., Lee, Y., \& Rey, S. 2003, ApJ, 591, L127

Quintana, H., Ramirez, A., \& Way, M. J. 1996, AJ, 111, 603

Stiavelli, M., Miller, B. W., Ferguson, H. C., et al. 2001, AJ, 121, 1385

Whitmore, B. C., Zhang, Q., \& Leitherer, C. 1999, AJ, 118, 1551

Worthey, G. 1994, ApJS, 95, 107 